\title{
Continuous Fabrication of Bio-Inspired Water Collecting Surface via Roll-Type Photolithography
}

\author{
Sung Ho Lee ${ }^{1, *}$, Jeong Hyeon Lee ${ }^{1, *}$, Cheol Woo Park', Choon Young Lee', Keesung Kim², \\ Dongha Tahk ${ }^{3}$, and Moon Kyu Kwak',\#
}

1 School of Mechanical Engineering, Kyungpook National University, Daegu, South Korea, 702-701 2 Institute of Advanced Machinery and Design, School of Mechanical \& Aerospace Engineering, Seoul National University, Seoul, South Korea, 151-742 3 Minuta technology Co., Ltd., Osan, South Korea, 447-210 \# Corresponding Author / E-mail: mkkwak@knu.ac.kr, TEL: +82-53-950-5573 *These authors equally contributed as corresponding author

KEYWORDS: Biomimetics, Continuous processing, Roll-to-roll processing, Water collection, Stenocara beetles

\begin{abstract}
We present a continuous fabrication method to make bio-inspired water collecting surface by using roll type photolithography for potential applications to real time air monitoring system. In this study, the carapace of the stenocara beetle was mimicked to achieve water collection from air, using a molding process involving micro-/nanofabrication techniques and roll type photolithography. We fabricated a super-hydrophilic surface on top of a super-hydrophobic surface and used two different setups to demonstrate water collection, a thermoelectric module and a humidifier. Also, the optimized geometric design for water collection was found from 16 different test samples. Detection of mercury is shown as a feasible practical application of such surfaces.
\end{abstract}

\section{Introduction}

In the field of micro-/nanotechnology, structural and surface properties such as adhesion, friction, optical characteristics, and wettability are very critical. Surfaces of biological organisms with such unique properties have been extensively studied and have been mimicked using various fabrication techniques to achieve myriad end applications. A few examples of biological surfaces with unique properties are the waterrepellency of a lotus leaf and directional wetting of spider webs; and a few examples of existing or possible end applications of mimicked biological surfaces are anti-reflective surfaces, dry adhesives, and locomotion of microrobots. ${ }^{1-7}$ In recent times, these biomimetic functional surfaces have been applied to the development of certain bio-related research areas and energy conversion systems such as cell environmental control systems, nanoelectric scaffolds, artificial skin, leaf-like photovoltaics, and autostereoscopic displays. ${ }^{8-12}$ Among these, the fabrication of synthetic waterrepellant surface is a very popular research area and is the closest to commercialization. With lotus leaf surfaces as inspiration, biomimetic super-hydrophobic surfaces have been synthesized; ${ }^{13-15}$ even superhydrophilic surfaces have been fabricated using different material retaining the same structure as super-hydrophobic. ${ }^{16,17}$ Various micro-/ nanofabrication methods have enabled realization of surfaces that mimic the lotus leaf, and in addition, several theoretical analyses have provided important cues for structural design. Various wetting phenomena were studied by many researchers in many ways. In previous fabrication method, Hybrid surface (hydrophobic and hydrophilic) was made by using wax or laser treatment. ${ }^{18,19}$ However, the patterned area using conventional fabrication method is restricted and not suitable for mass fabrication. So, it is desirable to find out a method for continuous fabrication of the surface that mimick stenocara beetle's back. ${ }^{20-25}$

In this study, bio-inspired water-collecting surfaces, made of a partially DUV(Deep ultraviolet or Ultraviolet C)-exposed prismatic micropattern, were employed to collect water vapor from the atmosphere. The carapace of the stenocara beetle was mimicked by fabrication of micro-/ nanostructures possessing optimal geometry using a molding process for water collection from ambient air. Stenocara is a species of the beetle that lives in Namib dessert of southern Africa. There are two types of stenocara's carapace. One is hydrophobic/hydrophilic combined surface and another is hydrophobic ridges on their carapace. ${ }^{26-28}$ In our study, we focused on complex wetting surface. Its carapace has hydrophilic parts and hydrophobic parts. And the beetle can survive by collecting water on the bumpy carapace surface from early morning fogs. ${ }^{27,28}$ To obtain optimal geometry for high water-collection efficiency, static water-contact angle and contact-angle hysteresis were predicted by the Cassie-Baxter and the 
Wenzel models. ${ }^{23,24}$ In the case of common bio-inspired water repellent surfaces, a higher water-contact angle and lower contact-angle hysteresis are essential for optimal surface performance. However, unlike in the case of the common lotus-like hydrophobic surface, low contact-angle hysteresis is not suitable for a water-collecting surface because the water droplets roll away before they get bigger by aggregating. So, hydrophilic and superhydrophobic combined surface is needed to have an adequate contact-angle hysteresis. To obtain optimized water-contact characteristics for a fabricated surface, various materials and micro-/nanogeometries were tested, and the water-collection process was demonstrated using the fabricated surface combined with a thermoelectric module. As proof of the feasible applicability of this surface, a small amount of mercury in the air was detected using the water collection device.

\section{Experimental Section}

\subsection{Materials}

UV-curable polyurethane functionalized with acrylate groups (PUA) was kindly provided by Minuta Tech. (Product name: MINS-301 RM, Korea)

\subsection{Continuous Fabrication of Prism-Shaped Micropatterns}

An adequate amount $\left(\sim 0.1-0.5 \mathrm{~mL} / \mathrm{cm}^{2}\right)$ of a UV-curable PUA polymer was drop-dispensed on a poly(ethylene terephthalate) (PET) film, and a roll-type prism-patterned master was carefully placed on top of the surface to make conformal contact. The PET film used in this study was surface-modified with urethane groups to increase adhesion to the acrylate-containing monome. The prism-patterned masters were provided by Minuta Tech. To cure, the film mold was exposed to UV $(250-400 \mathrm{~nm})$ at an intensity of $100 \mathrm{~mW} / \mathrm{cm}^{2}$.

\subsection{Continuous Fabrication of the Super-Hydrophobic/ Hydrophilic Complex Surface}

To enhance water-repellency, the surface of the replicated prism sheet was treated with vapor-phase trichloro $(1 \mathrm{H}, 1 \mathrm{H}, 2 \mathrm{H}, 2 \mathrm{H}-$ perfluorooctyl) silane. The prepared super-hydrophobic surface was placed on a roll-type DUV(F2151, EZ Lighting(Korea)) exposure system to make a super-hydrophillic surface on top of it. A cylindertype metal shadow mask with microscale patterns was used for blocking partially the DUV illumination at wavelengths of $254 \mathrm{~nm}$ and $185 \mathrm{~nm}$. In this process, Distance between mask and substrate is about $1 \mathrm{~cm}$ and DUV output is $58 \mu \mathrm{W} / \mathrm{cm}^{2}$. Although it takes long time ( $\sim 20$ mins) to change surface characteristic, but it can be shortened by using strong power of DUV source. After DUV exposure, the fabrication of super-hydrophobic/hydrophilic complex surface was completed.

\subsection{Water Collection Process using a Thermoelectric Module}

Water collection was conducted at room temperature and $70 \%$ humidity. To maintain constant temperature and humidity, an acrylic cage and a humidifier were used. For water vapor condensation, a thermoelectric module was used to chill the fabricated superhydrophobic/-hydrophilic complex surface down to dew point. Condensed vapor was collected in a vial for further experiments.
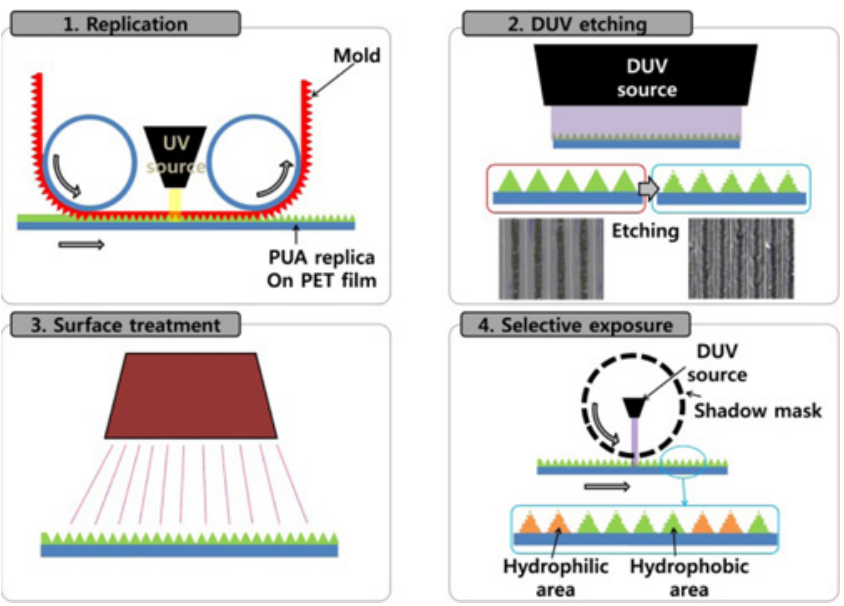

Fig. 1 Schematic illustration of continuous process for fabrication of bio-inspired water collection surface

\subsection{Water Contact Angle (CA) Measurement}

The static contact angle (CA) of water was measured with a contact angle analyzer equipped with a CCD camera (Drop Shape Analysis System DSA100, Kruss, Germany) by averaging values during 1.5 minutes for each pattern and the presented values were averaged again over at least six times of experiments with water volume of $5 \mu \mathrm{L}$.

\subsection{Mercury Detection}

Mercury-dissolved water was sprayed into the input section of the acrylic cage and water was collected on the opposite side of cage. Then, mercury was detected from the collected water using a Mercury Analyzer (CETAC M-7500, Parma Company(USA)).

\section{Result and Discussion}

Fig. 1 depicts an illustration of the experimental setup and flow of the continuous process used to fabricate water-collecting surfaces. The fabrication procedure consists of four detailed processes as follows: molding, DUV etching, surface treatment, and roll-type photolithography for selective exposure. Roll-to-roll UV-assisted patterning was performed with a commercially available prism-patterned sheet. Prior to continuous patterning, a polyurethaneacrylate (PUA) mold was replicated from a prism sheet by replica molding. To enhance the repellent property of the surface, the PUA surface was etched by deep UV illumination at wavelengths of $254 \mathrm{~nm}$ and $185 \mathrm{~nm}$. In this proof-of-principle experiment, a relatively weak DUV lamp $\left(58 \mu \mathrm{W} / \mathrm{cm}^{2}\right.$ of UV output) was used, and hence, it took approximately $8 \mathrm{~h}$ to complete the etching process. However, in principle, the continuous exposure method can be scaled up for fast fabrication to the order of a few centimeters per second of production speed. The deep-UV etching process and the related chemical analysis were reported earlier by Choi et al.In this previous research, the root-mean-square (RMS) roughness of fabricated surface was measured about $40 \mathrm{~nm}^{29}$ A bare PUA(non-pattern) surface has a water-contact angle of approximately $85^{\circ}$; while a much lower contact angle was measured for hierarchical patterns because Area between water and surface is larger than bare PUA. ${ }^{30}$ The hydrophilic surface, which has micro-/nanopatterns, temporarily maintained the Cassie- 


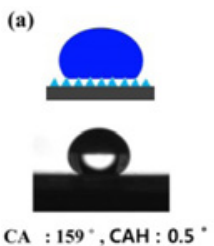

(b)

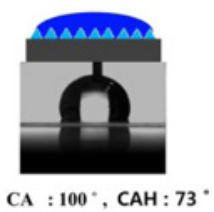

(c)

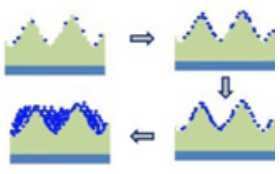

Fig. 2 (a) Water contact angle and contact angle hysteresis of water droplet which were collected against spray (b) Water contact angle and contact angle hysteresis of water droplet which were collected by condensation (c) Progress of water condensation on hierarchical patterns

Baxter state. However, the water-wetting state altered slightly from the Cassie-Baxter state to the Wenzel state owing to external vibrations or capillary forces. ${ }^{31}$ Therefore, patterned PUA surfaces need to be treated chemically to obtain high water-contact angles. Trichloro(3,3,3trifluoropropyl)silane was used for fabricating an anti-wetting surface coating using a vapor deposition method. The bare PUA substrate(nonpattern) with chemical surface-treatment recorded a contact angle of $110^{\circ}$. However, The hierarchical patterned substrate after the physical surface modification, the water-contact angle jumped to $159^{\circ}$, indicating a super-hydrophobic surface. To fabricate a super-hydrophobic/hydrophilic complex patterned surface, the hierarchically patterned surface was exposed in air to DUV illumination of wavelengths of 254 and $185 \mathrm{~nm}$. A roll-type shadow metal mask was used for the desired pattern. Deep-UV illumination caused the fluorine-terminated PUA surface to be photochemically altered to a polar, hydrophilic chain, with carbonyl species such as carboxylic acid at the surface.

Water collection experiments were performed in two different ways. In the first experiment, a fabricated sample was directly exposed to a mist of water. Throughout the water collection process, a hierarchically patterned surface was used, and the temperature was fixed at $25^{\circ} \mathrm{C}$. In the second experiment, water was collected by condensation. A thermoelectric module chilled down the surfactant-treated surface to dew point in $70 \%$ humidity and $25^{\circ} \mathrm{C}$ for $2 \mathrm{~h}$. In this case, a nonpatterned fluorine-terminated surface was used for water collection. Fig. 2 shows the wetting characteristics of the hierarchically patterned surface against two different water collection methods, and explains the need for two different types of surfaces used in the experiments. In general, a humidifier makes $5 \mu \mathrm{m}$-sized water droplets. These droplets are big enough to be suspended by the nanostructures on the microprism patterns, and the surface maintains a Cassie-Baxter state. As a result, a high static-water contact angle $\left(\sim 159^{\circ}\right.$ in both direction, across and along the line) and a low contact-angle hysteresis $\left(\sim 0.5^{\circ}\right)$ were measured. In the case of condensation, however, water particles wetted the ridges and valleys of the nanostructures owing to water vapor being much smaller compared to the pattern size, and therefore, a relatively low static-water contact angle $\left(\sim 100^{\circ}\right.$ in across the line, and $93^{\circ}$ in along the line $)$ and high contact-angle hysteresis $\left(\sim 73^{\circ}\right)$ were measured. This indicates that hierarchically-patterned surfaces are not suitable for the water collection process by condensation, even though they exhibit very high water-repellency. Finally, a non-patterned fluorine-terminated surface was used during the condensation process.

Fig. 4(a) shows the results on water collection with direct exposure to a mist. Mist drop size was around $10 \mu \mathrm{m}$, and mist flowed in

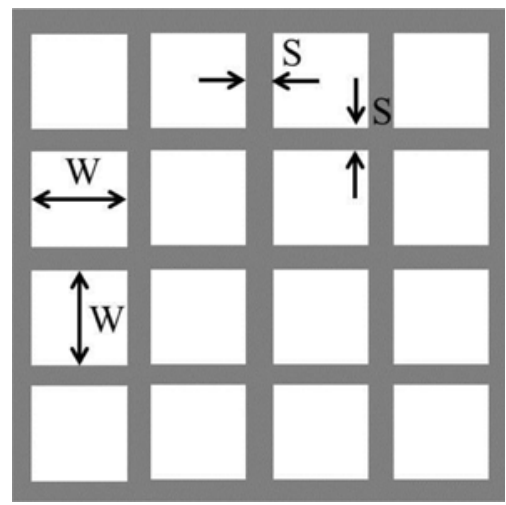

Fig. 3 The illustration of Metal mask used experiment. W is width and $\mathrm{S}$ is distance between patterns (a)

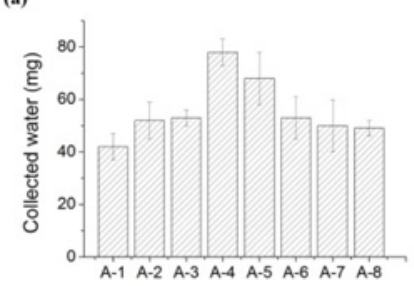

(b)

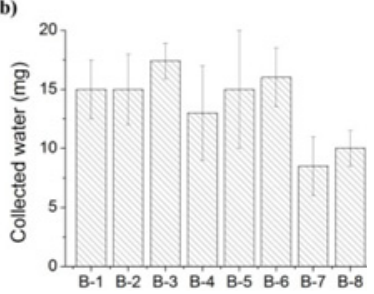

Fig. 4 (a) Amount of collected water by exposure method. Description of samples are shown in Table 1 (b) Amount of collected water by condensation. Description of samples are shown in Table 1

horizontal direction of water collecting surface. Including the hydrophobic and hydrophilic surfaces, eight different samples were used. Description for measured samples are shown in Table 1. The exposed hydrophilic area was determined with a clear field on a metal mask and two opening areas $(\mathrm{W} \times \mathrm{W} ; 3 \mathrm{~mm} \times 3 \mathrm{~mm}$ and $5 \mathrm{~mm} \times 5 \mathrm{~mm})$ were chosen. Distance(S) was also varied by $3 \mathrm{~mm}, 5 \mathrm{~mm}$, and $7 \mathrm{~mm}$ (Fig. 3).

A $3 \mathrm{~cm}$ by $3 \mathrm{~cm}$ sample was exposed for a duration of $3 \mathrm{~min}$. Fig. 4 shows an optimum combination ratio of the hydrophobic and hydrophilic surface. It was observed that the hydrophilic surface array with a width of $3 \mathrm{~mm}$ and a spacing of $5 \mathrm{~mm}$ on the hydrophobic surface gathered $77 \mathrm{mg}$ of water, and demonstrated a maximum water collection performance when compared with other cases. It was also easy to let the water droplets roll down because the hydrophobic area was relatively larger compared to the hydrophilic area.

Fig. 4(b) shows the results from the water collection experiments obtained using the condensation method. In contrast to the previous experiment with mist, no optimal conditions were obtained for water collection. The hydrophilic area was relatively larger compared to the first experiment since the water droplets grew growing from a very small size. The area of the specimen was $3 \mathrm{~cm}$ by $3 \mathrm{~cm}$, and it was chilled to $3^{\circ} \mathrm{C}$ in $70 \%$ of humidity and room temperature. As shown in Fig. 5(a), the temperature of the surface was chilled by thermoelectric module with a DC supply and water droplet was getting bigger until dripping.

Even though no regularity on the patterned geometry was observed, the amount of water collected was proportional to the time operated as shown in Fig. 5(b).

To demonstrate the feasibility of application of such surfaces, mercury detection from ambient air was studied. As shown in Fig. 6(a), 
Table 1 Description for samples dealt in Fig. 4

\begin{tabular}{|c|c|c|c|}
\hline Sample number in Fig. 4(a) & Description for samples in Fig. 4(a) & Sample number in Fig. 4(b) & Description for samples in Fig. 4(b) \\
\hline A-1 & Hydrophobic surface & B-1 & Hydrophobic surface \\
\hline A-2 & Hydrophilie surface & B-2 & Hydrophilie surface \\
\hline A-3 & $\mathrm{W}(3 \mathrm{~mm}): \mathrm{S}(3 \mathrm{~mm})$ & B-3 & $\mathrm{W}(0.5 \mathrm{~mm}): \mathrm{S}(0.5 \mathrm{~mm})$ Square array \\
\hline A-4 & $\mathrm{W}(3 \mathrm{~mm}): \mathrm{S}(5 \mathrm{~mm})$ & B-4 & $\mathrm{W}(0.5 \mathrm{~mm}): \mathrm{S}(1 \mathrm{~mm})$ Square array \\
\hline A-5 & $\mathrm{W}(3 \mathrm{~mm}): \mathrm{S}(7 \mathrm{~mm})$ & B-5 & $\mathrm{W}(0.5 \mathrm{~mm}): \mathrm{S}(1.5 \mathrm{~mm})$ Square array \\
\hline A-6 & $\mathrm{W}(5 \mathrm{~mm}): \mathrm{S}(3 \mathrm{~mm})$ & B-6 & $\mathrm{W}(0.5 \mathrm{~mm}): \mathrm{S}(0.5 \mathrm{~mm})$ Diagonal array \\
\hline A-7 & $\mathrm{W}(5 \mathrm{~mm}): \mathrm{S}(5 \mathrm{~mm})$ & B-7 & $\mathrm{W}(0.5 \mathrm{~mm}): \mathrm{S}(1 \mathrm{~mm})$ Diagonal array \\
\hline A-8 & $\mathrm{W}(5 \mathrm{~mm}): \mathrm{S}(7 \mathrm{~mm})$ & B-8 & $\mathrm{W}(0.5 \mathrm{~mm}): \mathrm{S}(1.5 \mathrm{~mm})$ Diagonal array \\
\hline
\end{tabular}
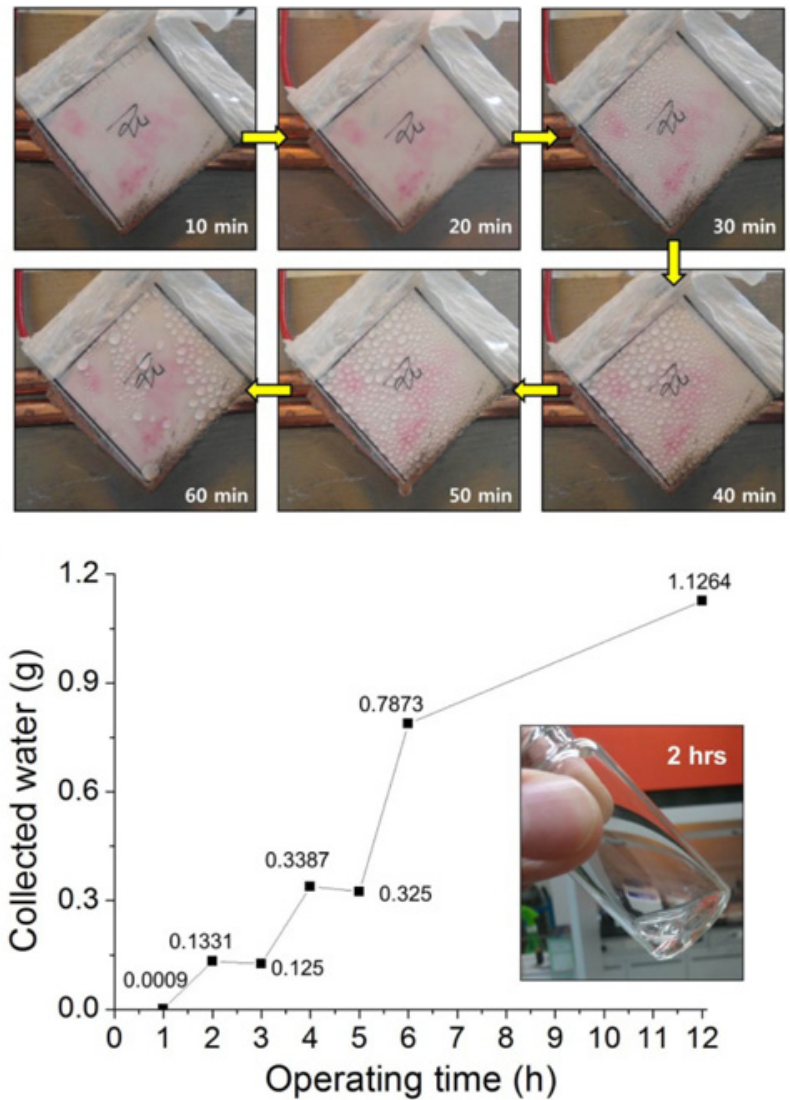

Fig. 5 (a) Time lapse image of water collection by using thermoelectric module (b) Progress of water collection as time passed

an acryl cage was designed for compulsory air flow, and water with soluble mercury was sprayed on the inside of the cage. The mixing ratio between mercury and water was 1 on 1000 (1000 ppm). Ambient air in the acryl cage was maintained at $70 \%$ humidity and $25^{\circ} \mathrm{C}$. Four sets of experiment were performed with various water collection times and doses of mercury, all at the same experimental conditions. Fig. 6(b) represents the amounts of mercury collected by the different experimental sets. From the results, it can be seen that the amount of collected mercury was proportional to the collection time and the initial dose of mercury. Although, there were some losses of injected mercury due to adsorption on the wall, approximately $3 \%$ of the initial input mercury was detected by analysis of the collected water. If air-flow and contaminant-injection systems are developed, sensitivity of the detection can be improved. Similar to mercury, many airborne contaminants, such as fine dust, tubercular bacillus, and other bacteria too, can be detected in real-time using this water collection system and additional sensory system. (a)

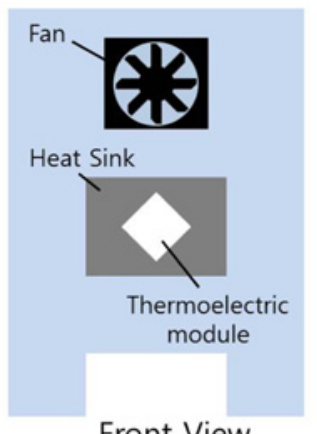

(c)

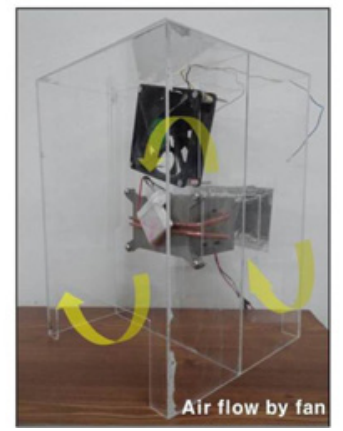

(b)

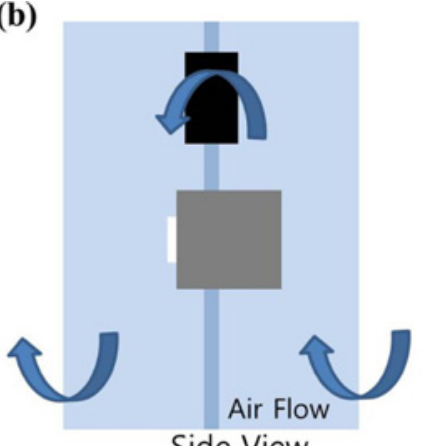

Side View

(d)

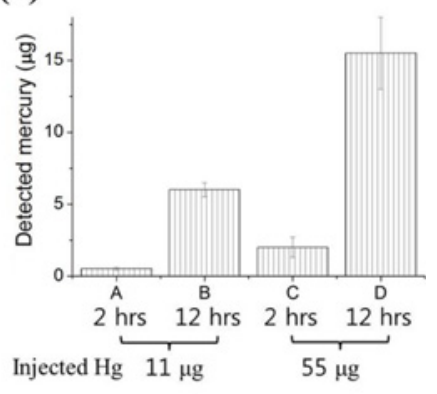

Fig. 6 The schematic illustration for experimental set up (a) Front view, and (b) Side view (c) Experimental set up for mercury detection. (d) Results of mercury detection experiments with four different experimental conditions

\section{Conclusion}

Through this study, a continuous fabrication process for bio-inspired water collection surfaces was established. Moreover, the performance of the water-collection surface was verified. Several optimization steps for the water collection surfaces, such as materials and optimal geometry, can possibly be performed in the future. The feasibility of water collection through two different ways was studied, and the feasibility of real-time detection of airborne contaminants was confirmed. In further research, these fabricated surfaces will be applied towards the detection of virus, bacteria, and other target materials by combining with microfluidic chip.

\section{ACKNOWLEDGEMENT}

The authors acknowledge support by a grant from the Young Researchers Supporting Program through the National Research Foundation of Korea (NRF) funded by the Ministry of Science, ICT 
and Future Planning (2012R1A1A1040040) and Global Excellent Technology Innovation (10045070), Small and Medium Business Administration (S2098429) funded by the Ministry of Trade, Industry\&Energy(MOTIE)/KEIT of Korea.

\section{REFERENCES}

1. Kwak, M. K., Jeong, H. E., Kim, T. I., Yoon, H., and Suh, K. Y., "Bio-Inspired Slanted Polymer Nanohairs for Anisotropic Wetting and Directional Dry Adhesion," Soft Matter, Vol. 6, No. 9, pp. 1849$1857,2010$.

2. Kwak, M. K., Pang, C., Jeong, H. E., Kim, H. N., Yoon, H., and et al., "Towards the Next Level of Bioinspired Dry Adhesives: New Designs and Applications," Advanced Functional Materials, Vol. 21, No. 19, pp. 3606-3616, 2011.

3. Menciassi, A., Accoto, D., Gorini, S., and Dario, P., "Development of a Biomimetic Miniature Robotic Crawler," Auton. Robots, Vol. 21, No. 2, pp. 155-163, 2006.

4. Sun, C. H., Jiang, P., and Jiang, B., "Broadband Moth-Eye Antireflection Coatings on Silicon," Applied Physics Letters, Vol. 92, pp. 061112-061113, 2008.

5. Zheng, Y., Bai, H., Huang, Z., Tian, X., Nie, F. Q., and et al., "Directional Water Collection on Wetted Spider Silk," Nature, Vol. 463, pp. 640-643, 2010.

6. Cho, K. J., Koh, J. S., Kim, S. W., Chu, W. S., Hong, Y. T., and Ahn, S. H., "Review of Manufacturing Processes for Soft Biomimetic Robots,” Int. J. Precis. Eng. Manuf., Vol. 10, No. 3, pp. 171-181, 2009.

7. Chu, W. S., Lee, K. T., Song, S. H., Han, M. W., Lee, J. Y., and et al., "Review of Biomimetic Underwater Robots using Smart Actuators,” Int. J. Precis. Eng. Manuf., Vol. 13, No. 7, pp. 12811292, 2012.

8. Dodgson, N. A., "Autostereoscopic 3D Display," Computer, Vol. 38, No. 8, pp. 31-36, 2005.

9. Pang, C., Lee, G. Y., Kim, T. I., Kim, S. M., Kim, H. N., and et al., "A Flexible and Highly Sensitive Strain-Gauge Sensor using Reversible Interlocking of Nanofibres," Nature Materials, Vol. 11, pp. 795-801, 2012.

10. Suh, K. Y., Park, M. C., and Kim, P., "Capillary Force Lithography: a Versatile Tool for Structured Biomaterials Interface Towards Cell and Tissue Engineering," Advanced Functional Materials, Vol. 19, pp. 2699-2712, 2009.

11. Tuzlakoglu, K., Bolgen, N., Salgado, A. J., Gomes, M. E., Piskin, E., and Reis, R. L., "Nano-and Micro-Fiber Combined Scaffolds: A New Architecture for Bone Tissue Engineering," J. Mater. Sci.: Mater. Med., Vol. 16, No. 12, pp. 1099-1104, 2005.

12. Wen, L., Hou, X., Tian, Y., Zhai, J., and Jiang, L., "Bio-Inspired Photoelectric Conversion Based on Smart-Gating Nanochannels,"
Advanced Functional Materials, Vol. 20, No. 16, pp. 2636-2642, 2010.

13. Lee, S., Oh, D., Jung, I., Bae, K., Jung, P., and et al., "Fabrication of Nickel Micromesh Sheets and Evaluation of Their Water-Repellent and Water-Proof Abilities,” Int. J. Precis. Eng. Manuf., Vol. 10, No. 3, pp. 161-166, 2009.

14. Kim, Y., Lee, J., Lee, I., Lee, S., and Ko, J., "Skin Friction Reduction in Tubes with Hydrophobically Structured Surfaces," Int. J. Precis. Eng. Manuf., Vol. 14, No. 2, pp. 299-306, 2013.

15. Barthwal, S., Kim, Y., and Lim, S. H., "Superhydrophobic and Superoleophobic Copper Plate Fabrication using Alkaline Solution Assisted Surface Oxidation Methods," Int. J. Precis. Eng. Manuf., Vol. 13, No. 8, pp. 1311-1315, 2012.

16. Jiang, L., Zhao, Y., and Zhai, J., "A Lotus-Leaf-Like Superhydrophobic Surface: A Porous Microsphere/Nanofiber Composite Film Prepared by Electrohydrodynamics," Angewandte Chemie, Vol. 116, No. 33, pp. 4438-4441, 2004.

17. Tahk, D., Kim, T. I., Yoon, H., Choi, M., Shin, K., and Suh, K. Y., "Fabrication of Antireflection and Antifogging Polymer Sheet by Partial Photopolymerization and Dry Etching," Langmuir, Vol. 26, No. 4, pp. 2240-2243, 2010.

18. Kim, T. I., Tahk, D., and Lee, H. H., "Wettability-Controllable Super Water- and Moderately Oil-Repellent Surface Fabricated by Wet Chemical Etching," Langmuir, Vol. 25, No. 11, pp. 6576-6579, 2009.

19. Nørgaard, T. and Dacke, M., "Fog-Basking Behaviour and Water Collection Efficiency in Namib Desert Darkling Beetles," Front. Zool., Vol. 7, Paper No. 23, 2010.

20. Young, T., “An Essay on the Cohesion of Fluids," Phil. Trans. R. Soc. London, Vol. 95, pp. 65-87, 1805.

21. Tadmor, R., "Line Energy and the Relation between Advancing, Receding, and Young Contact Angles," Langmuir, Vol. 20, No. 18, pp. 7659-7664, 2004.

22. de Gennes, P. G., "Wetting: Statics and Dynamics," Rev. Mod. Phys., Vol. 57, pp. 827-863, 1985.

23. Cassie, A. and Baxter, S., "Wettability of Porous Surfaces," Transactions of the Faraday Society, Vol. 40, pp. 546-551, 1944.

24. Wenzel, R. N., "Resistance of Solid Surfaces to Wetting by Water," Industrial \& Engineering Chemistry, Vol. 28, pp. 988-994, 1936.

25. White, B., Sarkar, A., and Kietzig, A. M., "Fog-Harvesting Inspired by the Stenocara Beetle - An Analysis of Drop Collection and Removal from Biomimetic Samples with Wetting Contrast," App. Surf. Sci., Vol. 284, pp. 826-836, 2013.

26. Dorrer, C. and Ruhe, J. R., "Mimicking the Stenocara BeetleDewetting of Drops from a Patterned Superhydrophobic Surface," Langmuir, Vol. 24, No. 12, pp. 6154-6158, 2008.

27. Parker, A. R. and Lawrence, C. R., "Water Capture by a Desert Beetle,” Nature, Vol. 414, pp. 33-34, 2001. 
28. Zhai, L., Berg, M. C., Cebeci, F. Ç., Kim, Y., Milwid, J. M., and et al., "Patterned Superhydrophobic Surfaces: Toward a Synthetic Mimic of the Namib Desert Beetle," Nano Letters, Vol. 6, No. 6, pp. 1213-1217, 2006.

29. Choi, S. J., Suh, K. Y., and Lee, H. H., "Direct Uv-Replica Molding of Biomimetic Hierarchical Structure for Selective Wetting," Journal of the American Chemical Society, Vol. 130, No. 20, pp. 6312-6313, 2008.

30. Kwak, M. K., Kim, T. I., Kim, P., Lee, H. H., and Suh, K. Y., "Large-Area Dual-Scale Metal Transfer by Adhesive Force," Small, Vol. 5, No. 8, pp. 928-932, 2009.

31. Jeong, H. E., Kwak, M. K., Park C. I., and Suh, K. Y., "Wettability of Nanoengineered Dual-Roughness Surfaces Fabricated by UVAssisted Capillary Force Lithography," J. Colloid Interface Sci., Vol. 339, No. 1, pp. 202-207, 2009. 